\title{
Pflegende werden Master
}

\author{
Uta Gaidys
}

Online publiziert: 30. November 2011

(C) Springer-Verlag 2011

Die Herausforderungen an das Gesundheitssystem verändern sich, werden komplexer und vielfältiger. Es gibt spezialisierte Versorgungsbedarfe von zum Beispiel chronisch erkrankten Menschen und/oder multimorbid erkrankten älteren und hochaltrigen Menschen. Damit hat sich das Aufgabenspektrum der Pflege verändert.

Die Profession der Pflege antwortet auf diese veränderten Bedarfe mit einem veränderten Qualifikations- und Bildungsziel. Es wurden Duale Bachelorstudiengänge an Hochschulen und Pflegeschulen eingerichtet. Die AbsolventInnen dieser Bachelor-Studiengänge sollen in der Lage sein, in unterschiedlichen Settings wie beispielsweise der ambulanten Pflege, der wohnortnahen Pflege, der Rehabilitation, in Akutkliniken oder in der stationären Langzeitpflege die patientennahe Pflegeversorgung zu übernehmen.

Mit diesen Studiengängen ist allerdings die Frage nach spezialisierten pflegerischen Bedarfen nicht beantwortet. International wurde deshalb eine sogenannte Advanced Nursing Practice eingeführt. Diese erweiterte und vertiefte pflegerische Praxis verändert die Rolle von Pflegenden. Pflegende werden Experten in spezialisierten Handlungsfeldern, treffen komplexe Versorgungsentscheidungen und verfügen über vertiefte klinische Versorgungskompetenzen. Die notwendige Bildungsqualifikation für diese pflegerische Praxis ist ein Masterabschluss.
Die Hochschule für Angewandte Wissenschaften Hamburg beginnt im Wintersemester 2012/2013 mit klinischen Kooperationspartnern einen Masterstudiengang, der Pflegende befähigt, im Sinne einer Advanced Nursing Practice in spezialisierten Handlungsfeldern wie der onkologischen/ palliativen Versorgung, der geriatrischen Pflege und der Intensivpflege tätig zu werden.
U. Gaidys $(\bowtie)$

Department Pflege und Management, Prodekanin Studium und Lehre, Fakultät Wirtschaft und Soziales, Hochschule für Angewandte Wissenschaften, Hamburg, Deutschland 